\title{
Laser Vaginal Rejuvenation
}

\author{
Amira Aziz ${ }^{1 *}$, Mohamed Amr El Noury ${ }^{2}$ and Tamer Taha ${ }^{3}$ \\ ${ }^{1}$ Assistant Researcher, Reproductive Medicine and Family Planning Department, Egypt \\ ${ }^{2}$ Professor of Obstetrics and Gynecology, Medical application of laser Sciences Department, Egypt \\ ${ }^{3}$ Assistant Professor, Reproductive Health and Family Planning Department, Egypt
}

*Corresponding author: Amira Aziz assistant Researcher, Reproductive Medicine and Family Planning Department, National Research Centre1, 33 el Bohous St. dokki, Cairo, 12311, Egypt.

Submission: 阱 February 19, 2018; Published: 阱 March 28, 2018

\begin{abstract}
We live in a society where beauty and sensations are important. Nowadays many women are concerned about their genital organs, especially the vaginal laxity associating vaginal deliveries and aging. Recently the market offers various modalities to treat such conditions, known as "Vaginal Relaxation". The aesthetic and functional procedures that comprise vaginal repair include traditional vaginal prolapse procedures as well as cosmetic vulval and labial procedures. The line between cosmetic and medically indicated procedures is blurred, and today many operations are performed for both purposes. Vaginal Rejuvenation (VR) is a new marketing term which is widely used to describe these vaginal procedures, but unfortunately with no clear specific procedure. Nowadays it describes the procedures aiming to "tighten" the vaginal canal and the introitus. However it mainly focuses on vaginal tightening to enhance sexual function and improve cosmetic appearance. In 2007, the American College of Obstetricians and Gynecologists became sufficiently concerned to issue a statement to practitioners warning that 'these procedures are not medically indicated and the safety and effectiveness of these procedures have not been documented'. One important potential problem is direct marketing to consumers rather than via referrals by health professionals. In our digital age, such marketing will rely heavily on the internet which is easily accessed by many women and young girls. More seriously, on the Internet particularly, medical experts now offer services and graphic details of the so called "vaginal rejuvenation". Unfortunately the quality and quantity of clinical information in vaginal rejuvenation provider sites is poor, with erroneous information in some instances. Method: We used the Google search engine and the single search term "Vaginal Rejuvenation", to obtain a broad data on this topic. Studies investigating and reviews on this issue were found. This Review defines VR, describes laser new technique for VR, collect evidences supporting this issue. Also it presents the experts' opinion regarding vaginal rejuvenation. Finally we tried to make a humble conclusion. Conclusion: Vaginal rejuvenation is one of the latest trends in elective vaginal procedure for women. However it needs more studies to investigate its efficacy and safety, instead as using it as a standard and routine procedure with known outcome (results and side effects). A proper exam is vital prior to any repair. Women seeking the vaginal rejuvenation should be educated about normal variants, evaluated for pelvic support disorders and screened for psychological conditions. When dealing with sexual dysfunction alone and the caliber or width of the vagina, repair must be very meticulous and exact in order enhance sensation and function and not impair it.
\end{abstract}

Keywords: Vaginal rejuvenation; Laser vaginal rejuvenation; Vaginoplasty; Vaginal tightening

\section{Mini Review}

\section{Historical background}

Vaginal rejuvenation or tightening is over thousand year old. In $1050 \mathrm{AD}$, the female physician Trota de Ruggiero from Salerno Italy mentioned in her work De Curis Mulierum (Treatments for women) five nonsurgical recipes for "restoring virginity". Later she contributed to another work De Ornatu Mulierum (Women's Cosmetics), which in addition to two other works written by other medieval physicians comprised the compendium on women's medicine commonly known as the Trotula. The Curis Mulierum ensemble became the most widely disseminated and translated works on women's medicine in later medieval Europe. The techniques described in Trotula to suture vaginal lacerations at birth forms the basis for all modern vaginoplasty procedures [1].

In 2002 David Matlock an Ob and Gyn in Beverly Hills started offering vaginoplasties to enhance sex. He trademarked his style of vaginoplasty in newspaper ads. Then he developed a franchise model for others to learn the procedures using a trademarked terminology. He started this business with heavy advertising in journals and in meetings. Meanwhile the cosmetic surgery exploded in popularity worldwide, owing to the introduction of reality TV, Botox, credit card market and social media.

Since then, the market starts to offer a great variety of options for vaginal rejuvenation to the women concerning about their genitalia. In addition with the worldwide use of the internet, many cosmetic centers are offering such treatment modalities which become now accusable to women and young girls and recently has invaded the Middle East market [2].

\section{Now a days}

With the availability of this media and huge market, many women become now aware of the options offered to enhance 
their beauty. Sexual activity plays a major role in the women selfconfidence. Therefore vaginal health shares an important part of the female beauty. This issue is of a great importance especially in our society, the Middle East region, where vaginal deliveries are still preferred especially in the rural areas. Also due to the spread of poverty, sexual activity seems to be one of the biggest source of satisfaction in our population. It is now widely accepted that normal vaginal delivery is the major predisposing factor for the loss of normal vaginal tightness recently known as vaginal relaxation syndrome (VRS) [3]. Loss of vaginal tightening is also caused by aging due to hormonal changes related to the menopause, as well as chronic constipation, cough and overweight [4]. Women visit a gynecologist, with loose vagina complaining of decreased friction during intercourse leading to reduction in sexual gratification, either by herself, or by her partner.

Therefore VRS becomes a rising but a hidden problem in our society, since these women find it a shame to speak upon their sexual life, even if they have a problem. Actually a relation between vaginal relaxation and sexual dysfunction has been documented in many studies [5]. In addition vaginal relaxation may be one of the earlier symptoms before an obvious pelvic organ prolapsed (POP) becomes symptomatic [6]. POP in turn may result in urinary, fecal incontinence and/ or urge incontinence, which may deteriorate the patient quality of life. A large number of treatment modalities are available for such condition. Kegel exercise and locally administered tightening cream, local or systemic estrogen, which may be contraindicated in some women; despite their safety, have limited efficacy and requires long term commitment. Vaginoplasty/ Vaginal Rejuvenation is now widely accepted to be the alternative long term treatment for these conditions [7].

Accordingly the objective of this paper is to collect the available data describing Vaginal Rejuvenation with a focus on the Laser therapy, reviewing the literature discussing the techniques used and their results, in a humble trial to collect evidences regarding this newly emerging topic. Search was performed using Google search engine, with these keywords: vaginal rejuvenation, vaginal tightening, vaginoplasty, laser vaginal rejuvenation. The majority of the reviewed papers are published during the last six years.

\section{Vaginal Rejuvenation}

\section{Definition}

Vaginal Rejuvenation (VR) is a new ongoing market term used recently by many cosmetic centers around the world; to describe the vaginal surgical procedures. Unfortunately it has with no clear specific procedure. However it is used nowadays to describe the procedures aiming to "tighten" the vaginal canal and the introits. Actually it is not a new technique; it seems to be a modification of the traditional vaginal reconstructive surgeries aiming to correct the vaginal wall prolapse [7]. This term can be interpreted as vaginoplasty, perineorrhaphy, labiaplasty, colpoperineorrhaphy... etc.; there is an overlap between the different procedures. However it mainly focuses on vaginal tightening to enhance sexual function and improve cosmetic appearance. The efficacy of such procedures on sexual functions was intensively investigated. Still this is not totally accepted to operate only to treat VRS, being a surgical procedure it carries the risk of complications leading to scarring, impairing nerve and blood supply resulting in either dyspareunia or decrease sexual sensation [3].

\section{Types}

The Vaginal Rejuvenation can be divided according to the present treatment options into:

i. $\quad$ Reconstructive VR

ii. Cosmetic VR

iii. Functional VR [8].

i. Reconstructive vaginal rejuvenation

This type of procedure includes all surgeries aiming to repair and/or correct the anatomical vaginal structures. They are mainly the well-known vaginal surgeries which correct the pelvic organ prolapse and the incontinence (anterior, posterior colporrhaphy, hysteropexy, urethral sling and anal sphincteroplasty), decrease a wide vaginal diameter (perineoplasty) following vaginal deliveries or aging process, repair a birth defect after vaginal delivery, correct the congenitally malformed vagina (vaginoplasty) e.g. agenesis, septum...etc [5].

\section{ii. Cosmetic vaginal rejuvenation}

These procedures aim to alter the appearance of the vulva and vaginal opening. They include: Labioplasty: in the form of: restoring symmetry, reducing or removing inner labia, removing of unwanted fat from the mons pubis and upper parts of the labia majora, liposculturing, augmentation labiaplasty. Revirginization: hymen repair, hymenoplasty. Hoodectomy: reducing clitorial hood. Vaginal Rejuvenation: shortening and tightening the vaginal canal and introitus, by removing unwanted redundant mucosa and skin [7].

\section{iii. Functional vaginal rejuvenation}

These procedures focus mainly on enhancing sexual sensation. They include: Clitorial Unhooding: A lead to increase clitorial stimulation thus intensifies sexual pleasure. G-Spot amplification: making it larger and more sensitive through injecting collagen. Vaginal Rejuvenation: tightening a wide vaginal canal and introitus [5].

As we can notice from the above mentioned procedures, we can observe that the "Vaginal Rejuvenation" provides cosmetic and functional treatments, but not a reconstructive treatment. Therefore we can suggest that Vaginoplasty is a reconstructive plastic surgery for the vaginal canal and vulva that might be damaged due to congenital (e.g. septum) or acquired (e.g. childbirth trauma) causes. However Vaginal Rejuvenation is a non-reconstructive vaginal surgeries aim to tighten the vagina, by removing excessive 
mucosa and tightening the supportive structures caused by aging or parturition, without the presence of a clear medical indication.

\section{Laser Vaginal Rejuvenation}

\section{Background}

For a long time ago, Laser treatment has been used safely and effectively in many medical fields like dermatology and dentistry. The idea of using the ability of laser energy to restore and regenerate damaged tissue provides a new ways called "Regenerative Medicine". With the introduction of minimally ablative fractional laser, the gynecologist started to apply this new laser technique to treat common vaginal and vulval disorders. In 2014, the Italian DEKA company's SmartXide2 CO2 laser technology, were cleared by the US Food and Drug Administration (FDA) for its use in various medical fields e.g. dermatology, plastic surgeries, otolaryngology, genitourinary surgery etc [9]. Since the FDA release, the market started to offer a great variety for laser technology to the healthcare professionals as well as directly to the consumer.

In the last few years, many gynecologists were investigating the possible use of laser for the treatment of different gynecological conditions e.g. Genitor-urinary syndrome of menopause, vaginal relaxation syndrome and mild cases of stress incontinence. All of these procedures are gathered in the marketing term, known as "Laser Vaginal Rejuvenation".

\section{Types of lasers used: (Table 1)}

Table 1: Differences between $\mathrm{CO}_{2}$ and Er: YAG lasers in the treatment of genitourinary syndrome of menopause (GSM) (A. Gaspar, as presented at FIGO 2012, personal communication).

\begin{tabular}{|c|c|c|}
\hline & $\mathrm{CO}_{2}$ Laser & Erbium: YAG Laser \\
\hline Absorption in water & 15x less than Erbium: YAG & $15 x$ more than $\mathrm{CO} 2$ \\
\hline Optical penetration & $50 \mu \mathrm{m}$ & $3-5 \mu \mathrm{m}$ \\
\hline Mechanism of action & Ablation & Thermal diffusion \\
\hline Adverse effects & Always partial necrosis & Mucosa is not damaged \\
\hline Depth of penetration & $3 \mathrm{~mm}$ or more & $200-500 \mu \mathrm{m}$ \\
\hline Pain during treatment on scale $0-10$ & 5 & 0 \\
\hline Pain post-treatment on scale $0-10$ & 5-Mar & 0 \\
\hline Treatment area & Vagina & Vaginal canal and introitus \\
\hline Tissue healing phase (days) & 20 & 2 \\
\hline Return to normal sexual activity (days) & 10 & 3 \\
\hline Laser release & Operator-dependant & Uniform and controlled \\
\hline Advantages & More effective & More safe \\
\hline Disadvantages & Increase Residual Thermal Damage (RTD) & Is it Effective?! \\
\hline Brand names & Monalisa smile, Femilift.. & Intimalase, Asclepion.. \\
\hline
\end{tabular}

\section{Committee opinions}

It was for the first time in 2007, that the vaginal cosmetic procedures were addressed by means of a Committee Opinion. The American College of Obstetrics and Gynecology issued a warning, addressing directly David Matlock practice. They stated that his exact procedures are not clear and they have no medical indications.
Nowadays "Laser Vaginal Rejuvenation" (LVR) is gaining a great popularity from patients and physicians. Laser is known for its lesser side effects compared to surgery being an outpatient procedure. So it is very appealing to treat the above mentioned conditions, but is it as efficient as surgery? Surprisingly practice of LVR dated before studying its efficacy and safety. Unfortunately, there is no solid evidence supporting its efficacy and safety till now [10]. No one can deny the big participation of David Matlock, the gynecological and plastic surgeon in West Hollywood, California, who is a prominent promoter of this LVR technique. In 2006, He established the Laser Vaginal Rejuvenation Institute of America [11].

\section{Definition}

It is the use of laser photothermal effect on vaginal mucosa in order to tighten a lax/wide vaginal canal and introitus.

\section{Mechanism of action}

i. Stimulation of collagen remodeling, resulting in new collagen regeneration, which leads to vaginal wall thickening with the end result of vaginal tightening.

ii. Shrinkage of the endow-pelvic fascia, ligament and mucosa of vaginal wall to improve stress urinary incontinence [12]. 
in female genital cosmetic surgeries provider sites is poor with erroneous information in some instances. Impeccable professionalism and ethical integrity is crucial for this controversial practice. Clear and detailed guidelines on how to raise the standard of information to women on all aspects of FGCS are urgently needed"[14].

Recently in 2013, the Society of Obstetrics and gynecology of Canada published a policy statement in the journal of obstetrics and gynecology Canada. They came out with recommendations, they mainly focus on: Education and proper counseling of the women requesting these procedures, explaining the individual genitalia variations; proper and full medical, sexual and gynecological history is mandatory for proper patients selection; Physicians providing these techniques should clarify that these were not result in improving sexual function and finally the non-medical terms used such as vaginal rejuvenation, must be recognized as marketing term with no medical indications [15].

\section{Studies}

Despite this vigorous attack on the vaginal rejuvenation, the health professionals working in the genitor-urinary as well as the plastic surgeons are still convinced of the promising treatment that laser can offer for many genitor-urinary disorders. Therefore recently, many researches started to

Investigate the efficacy and safety of laser technology for treating vast gynecological conditions. Many studies were then performed examining the two different types of laser used, $\mathrm{CO} 2$ and Erbium: YAG lasers, in treating common vaginal problems, genitor-urinary syndrome of menopause (GSM), vaginal relaxation syndrome (VRS), menopausal vaginal atrophy, mild degree of stress urinary incontinence. They assessed the results using a various methods, starting from visual analog to vaginal biopsy.

\section{Carbone dioxide laser}

Surprisingly even before the FDA approval of the CO2 laser use in gynecology, DEKA company has introduced the new operating mode to treat vaginal disorders, the D-pulse or Deka Pulse in the SmartXide V2LR machine. D pulse consists of two parts: the initial high power peak which allows superficial ablation of the vaginal epithelium of the atrophic mucosa, then the second part is a low power longer duration pulse, which allows deep penetration, in order to reach the matrix components i.e. collagen, fibril and blood capillaries [16]. Many studies were then conducted, examining its efficiency in treating mainly vaginal atrophy related to menopause. One of the earliest studies using this laser mode was done by Gasper and colleagues [17]. The study composed of two groups, study group and control group. The study group was subjected to fractional CO2 laser, platelets rich plasma (PRP) and pelvic exercise; while the control group had only PRP and pelvic exercise. Their results showed significant improvement of the vaginal dryness, discomfort during intercourse. On the histological level, the vagina showed increase in the fibrillar component of the extracellular matrix, neogenesis in addition to increase thickness of the vaginal epithelium. However, some complications were recorded such as mild vaginal bleeding, pain and burning sensation during or even after the treatment. Later in 2013 Salvatore and Calligaro [18] examined the effect of SmartXide2 V2LR system not only on vaginal atrophy, but also on the vaginal laxity and urinary incontinence. Their study showed improvement of the Visual analoge scale for both vaginal atrophy as well as urinary incontinence. Salvatore had done another pilot study in 2014 [19], proving the efficiency of fractional $\mathrm{CO} 2$ in relieving dyspareunia caused by vaginal atrophy. Lately in 2015, he did a very interesting study testing this CO2 laser treatment on ex vivo vaginal specimens from postmenopausal women. This study resulted in tissue remodeling without damage of the surrounding tissue [20]. Despite the promising results of the above mentioned studies, they are considered weak, due to the small sample sizes and lack of long term follow up.

\section{Erbium: YAG laser}

Erbium:YAG laser is known for its non-ablative thermal only effect on the irradiated tissue. Being a second generation laser, it begins to gain attention of the medical community to replace the CO2 laser for treatment of vaginal disorders. Erbium: YAG is much more absorbed by water, making its

penetration less than CO2. Actually Gasper, who had first showed interest in fractional CO2 laser in 2011, had done a recent study. In 2013, Gasper compared the efficacy and safety of ablative CO2 laser with the new non-ablative Erbium YAG laser procedure for treatment of vaginal atrophy. Each group consisted of 35 patients, who were evaluated with sexual questionnaire, vaginal cytology and biopsies. His study demonstrated that Erbium YAG laser procedure resulted in better results was well tolerated, and less invasive than the $\mathrm{CO} 2$ procedure, with lesser complications as compared with the complications observed in the $\mathrm{CO} 2$ treated group such as pain and burning sensation [21].

As a market competition, the Erbium YAG laser system companies begin to provide laser system specially designed for the vaginal tightening (The Intimalase). In addition, the medical society finds this more seductive to use to prevent $\mathrm{CO} 2$ laser complications. Since then, many studies are done testing its efficacy and safety for treating vaginal disorders. Gaviria and Lanz (2012) investigated Erbium YAG laser therapy for treating vaginal relaxation syndrome. This was only a pilot study including 21 patients, for whom subjective and objective assessments were done to assess treatment success. Pelvic organ prolapse quantification system showed significant improvement of prolapse; laser vaginal tightening questionnaire, was also tailored to assess patient satisfaction, showed improvement of vaginal tightness [3]. However this was only a pilot study that included patients with different vaginal disorders: vaginal laxity, prolapse and urinary incontinence. In the same year Vinzintin and colleagues had conducted a multi-centre study. They analyzed a special Er: YAG Pixel Screen technology with the smooth mode concept that has been introduced by the manufacturer Fotona. Fotona offered two techniques the Intimalase and Incontilase to treat vaginal relaxation and urinary incontinence respectively. The Smooth Mode technology entails the delivery of fast sequence low fluence laser pulses inside an overall super long 
pulse. As a consequence, the laser energy is transmitted as heat on the mucosal surface without resulting in ablation, and spread deeply into vaginal mucosa. Their results were promising and the suggested that this might be a minimally invasive solution for many vaginal problems [22]. Fotona manufacturer didn't stop; they came with two scanning scopes $\left(360^{\circ}\right.$ and $90^{\circ}$ scopes), they differ in the shape of laser beam and the spot size of laser onto the tissue. A comparative study in 2014 was done by Lee, to test which scope do better with lesser complications [23].

In the last two years, Er: YAG laser was also examined to treat Genito-urinary syndrome of menopause. Since this syndrome was the first problem to be treated using the CO2 laser. In 2015 Gambacciani and colleagues showed significant decrease in both vaginal dryness and dyspareunia in addition to an increase in the Vaginal Health Index Scoring system (VHIS). Despite that it was a pilot study, however it is one of the first studies with follow up for 24weeks. Fortunately it showed sustained beneficial results [24]. This study results encourages a group of physicians from Italy in 2015 to establish the first Vaginal Erbium Laser Academy (VELA) They presented at the first International Focus Meeting of the VELA the first multi-centre large scale VELA study designed to evaluate laser treatment on 1500 patients. Findings from their study are promising till now. They suggested that laser energy enhances the collagen synthesis and remodeling in the vaginal mucosa [12]. Last but not least, Gaviria and colleagues in 2016 had published a study which is prominent with its 3 year follow up duration. They focused mainly on patient satisfaction assessment, for which they Formulated a special laser vaginal tightening questionnaire after 3years follow up, in $87.5 \%$ of Patients the results persist, in $58 \%$ of all participants expressed high satisfaction, $83.3 \%$ willing to Repeat the therapy, while $90 \%$ would recommend the treatment [25].

\section{Conclusion}

Laser vaginal rejuvenation is an emerging accessible technique that we cannot avoid. Therefore it Become the medical community responsibility to examine its efficacy and safety. Physicians dealing With these types of problems should do proper patient selection should be aware of the limitations of LVR, and be ready for any complications to manage properly, In addition to put a follow up schedule to ensure the life-long effect on the female genital organs some health care professionals perform. Such procedures only upon females request; however a clear explanation of the procedure with the Potential adverse effect should be clarified to every woman before an action, plus a clear indication should be identified in the actual life, it is offered as a routine safe treatment for many vaginal problems In fact, anatomical restoration is not always accompanied by improvement in bladder or Sexual function; their impact on the patient quality of life should be of major concern after such LVR Procedure that is why in many studies, a subjective assessment of the treatment is also an important Measure to evaluate LVR. Since this treatment involves the female sexual organ, it is logic to think that it should affect positively her sexual life without impairing her sexual activity or interfering with quality of life.

\section{References}

1. Green MH (2001) The Trotula: an English translation of the medieval compendium of women's medicine. University of Pennsylvania Press, pp. 248.

2. Pelosi M (2015) Cosmetic vaginal surgery: a review.

3. Gaviria J, Lanz J (2012) Laser vaginal tightening (LVT)-evaluation of a novel noninvasive laser treatment for vaginal relaxation syndrome. Journal of the Laser and Health Academy 2012(1): 59-66.

4. Abedi P, Jamali S, Tadayon M, Parhizkar S, Mogharab F (2014) Effectiveness of selective vaginal tightening on sexual function among reproductive aged women in Iran with vaginal laxity: a quasiexpermental study. J Obstet Gynaecol Res 40(2): 526-531.

5. Moore R, Miklos J, Chinthakanan O (2014) Vaginal Reconstruction/ Rejuvenation: is there data to support improved sexual function? An Update and Review of literature. Surg Technol Int 25: 179-190.

6. Di Tonna F, Mazzariol C, Optale G, Piazza N, Pianon C (2009) Evaluation of female sexual function after vaginal surgery with the FSFI (Female Sexual Function Index): our experience. Journal of Andrological Sciences 16: 57-61.

7. Iglesio CB, Yurteri Kaplan L, Alinsod R (2013) Female genital cosmetic surgery: a review of techniques and outcomes. Int Urogynecol J 24(12): 1997-2009.

8. Romanzi L (2010) Vaginal Rejuvenation Defined.

9. Hutchinson J, Segal S (2015) Genitourinary Syndrome of menopause and the use of laser therapy. Maturitas 82(4): 342-345.

10. Gambacciani M, Cervigni M (2015) Erbium laser in gynecology: aims, inspirations and action points. Climacteric 18(1): 2-3.

11. The Intimate Rejuvenation and Innovative Surgery Center (IRIS) establishes the laser vaginal rejuvenation institute (R) of Arizona.

12. Gambacciani M, Torelli MG, Martella L, Bracco GL, Casagrande AG, et al. (2015) Rationale and Design for the Vaginal Erbium Laser Academy Study (VELAS): An international multicenter observational study on genitourinary syndrome of menopause and stress urinary incontinence. Climacteric 18(1): 43-48.

13. Committee on Gynecologic Practice, American College of Obstetricians and Gynecologists (2007) ACOG Committee Opinion No. 378: Vaginal "Rejuvenation" and Cosmetic Vaginal Procedures. Obstet Gynecol 110(3): 737-738.

14. Lih Mei L, Taghinejadi N, Creighton SM (2012) An Analysis of the content and Clinical Implications of online Advertisements for Female Genital Cosmetic Surgery. BMJ Open 2(6): e001908.

15. The Sociaty of Obstetrics and Gynecology of Canada Policy Statement (2013) Female Genital Cosmetic Surgery. No.300.

16. (2012) D-pulse: the pulse created for Monalisa Touch.

17. Gasper A, Addamo G, Brandi H (2011) Vaginal Fractional CO2 laser: a minimally invasive option for vaginal rejuvenation. The American Journal of Cosmetic Surgery 28(3): 156-162.

18. Salvatore $\mathrm{S}$, Calligaro A, (2013) Use of $\mathrm{CO}_{2}$ laser therapy against Vaginal Atrophy, Vaginal Laxity and Urinary Incontinence. Deka White Paper.

19. Salvatore S, Nappi RE, Zerbinati N Calligaro A, Ferrero S, et al. (2014) A 12 week treatment with fractional $\mathrm{CO}_{2}$ laser for vulvovaginal atrophy: A pilot study. Climacteric 17(4): 363-369.

20. Salvatore S, Leone U, Athanasiou S, Origoni M, Candiani M (2015) Histological Study on the effect of microablative fractional $\mathrm{CO}_{2}$ laser on atrophic vaginal tissue: an ex vivo study. Menopause 22(8): 845-849.

21. Gasper A (2013) Evolution of Minimally Invasive Laser Treatment for Vaginal Atrophy. Journal of the Laser and Health Academy (1): S28. 
22. Vinzintin Z, Rivera M, Fistonic I, Sarcoglu F, Guimares P et al. (2012) Novel Minimally Invasive VSP Er: YAG Laser treatment in Gynecology. Journal of the Laser and Health Academy (1).

23. Lee MS (2014) Treatment of Vaginal Relaxation Syndrome with an Erbium:YAG Laser using $90^{\circ}$ and $360^{\circ}$ Scanning Scopes: A pilot Study \& short-term Results. Laser Ther 23(2): 129-138.
24. Gambacciani M, Levancini M, Cervigni M (2015) Vaginal erbium laser: the second generation thermotherapy for the genitourinary syndrome of menopause. Climacteric 18(5): 757-763.

25. Gaviria J, Korosec B, Fernandez J, Montero G (2016) Up to 3 year followup of patients with vaginal relaxation syndrome participating in laser vaginal tightening. Journal of the Laser and Health Academy. (c) $\begin{aligned} & \text { Creative Commons Attribution } 4.0 \\ & \text { International License }\end{aligned}$

For possible submissions Click Here

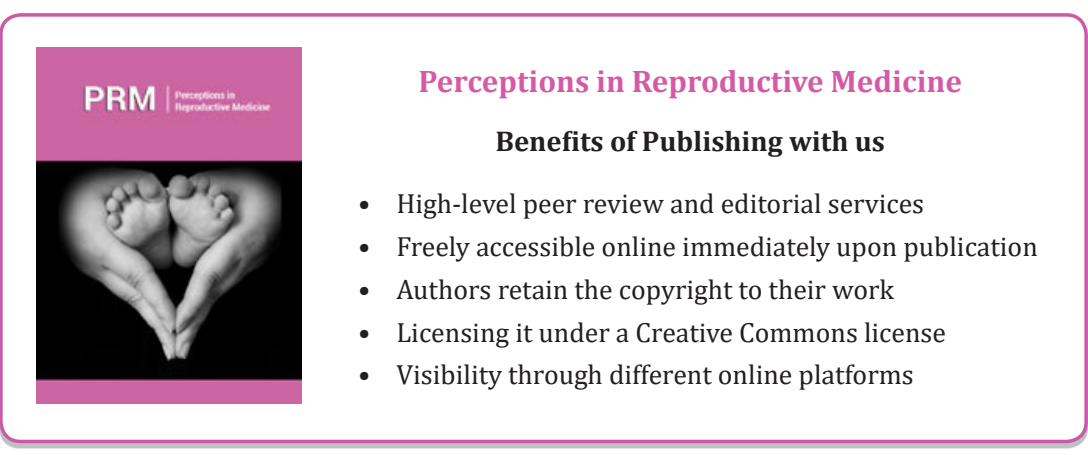

